\title{
Molecular epidemiology of hepatitis E virus infections in Shanghai, China
}

Yumin Zhu ${ }^{1,2}$, Fusheng $\mathrm{Si}^{1,3}$, Dianjun $\mathrm{CaO}^{4}$, Xiaoming $\mathrm{Yu}^{1,3}$, Ruisong $\mathrm{Yu}^{1,2}$, Shijuan Dong ${ }^{1,2}$, Fenfen Huang ${ }^{1,5}$, Yuanshu Zhang ${ }^{3}$ and Zhen $\mathrm{Li}^{1 *}$

\begin{abstract}
Background: Hepatitis E virus (HEV) causes acute or fulminant hepatitis in humans and is an important public health concern in many developing countries. China has a high incidence of HEV epidemics, with at least three genotypes (1, 3 and 4 ) and nine subtypes (1b, 1c, 3b, 4a, 4b, 4d, 4g, 4h and 4i) so far identified. Since genotype 3 and the newly identified subtype $4 i$ have been exclusively limited geographically to Shanghai and its neighboring provinces, the epidemiology of HEV infections within the municipality, a major industrial and commercial center, deserves closer attention.

Findings: A total of 65 sequences, 60 located within the HEV SH-SW-zs1 genome [GenBank:EF570133], together with five full-length swine and human HEV genomic sequences, all emanating from Shanghai, were retrieved from GenBank. Consistent with the primary role of genotype 4 in China overall, analysis of the sequences revealed this to have been the dominant genotype (58/65) in Shanghai. Six HEV subtypes (3b, 4a, 4b, 4d, $4 \mathrm{~h}$ and $4 \mathrm{i}$ ) were also represented. However, although subtype $4 a$ is the dominant subtype throughout China, subtype 4i (29/65) was the most prevalent subtype among the Shanghai sequences, followed by subtypes $4 d(10 / 65)$ and $4 \mathrm{~h}(9 / 65)$. Subtypes $4 \mathrm{~h}, 4 \mathrm{i}$ and $4 \mathrm{~d}$ were found in both swine and humans, whereas $4 \mathrm{~b}$ was found only in swine and subtype $4 \mathrm{a}$ only in humans.
\end{abstract}

Conclusions: Six different swine and human HEV subtypes have so far been documented in Shanghai. More molecular epidemiological investigations of HEV in swine, and particularly among the human population, should be undertaken.

Keywords: Hepatitis E virus, Epidemiology, Shanghai municipality, Virus genotypes, Virus subtypes

\section{Findings}

Hepatitis E virus (HEV), the causative agent of acute or fulminant hepatitis in humans, is an important public health concern in many developing countries. It is estimated that about two billion people, or one-third of the world population, live in areas where HEV is endemic and are therefore at risk of infection [1]. The disease is thought to be transmitted by the fecal-oral route, usually through contaminated drinking water.

HEV is a non-enveloped, single stranded, positivesense RNA virus belonging to the family Hepeviridae. At least four mammalian HEV genotypes have been

\footnotetext{
* Correspondence: zhenli60@163.com

'Institute of Animal Science and Veterinary Medicine, Shanghai Academy of Agricultural Sciences, Shanghai 201106, China

Full list of author information is available at the end of the article
}

recognized [2]. Genotypes 1 and 2 are primarily associated with fecal-oral transmission among humans and, in developing countries, can lead to waterborne jaundice epidemics. Genotypes 3 and 4 circulate in humans and several animal species, and are associated with sporadic infections among humans in industrialized countries [3]. In addition, two putative HEV genotypes, one from the Norway rat (Rattus norvegicus) [4] and the other from wild boar [5], were recently reported.

A recent study in China, where there is a high frequency of HEV epidemics, has shown that HEV seroprevalence among the general population was almost $40 \%$ and increased with age at a rate of about $1 \%$ per year [6]. Furthermore, the number of fecal samples taken from young swine that tested positive for HEV RNA ranged between $20-48 \%$ [7,8]. According to Zhu et al.
Ciomed Central

() 2011 Zhu et al; licensee BioMed Central Ltd. This is an Open Access article distributed under the terms of the Creative Commons Attribution License, which permits unrestricted use, distribution, and reproduction in any medium, provided the original work is properly cited. 
[9], at least three genotypes (1, 3 and 4$)$ and nine subtypes $(1 \mathrm{~b}, 1 \mathrm{c}, 3 \mathrm{~b}, 4 \mathrm{a}, 4 \mathrm{~b}, 4 \mathrm{~d}, 4 \mathrm{~g}, 4 \mathrm{~h}$ and $4 \mathrm{i})$ of human and swine HEV have so far been documented in China. Since genotype 3 and the newly identified subtype $4 \mathrm{i}$ have been limited geographically exclusively to Shanghai and its neighboring provinces, the municipality, as a major industrial and commercial center, is deserving of close attention with respect to HEV infection epidemiology.

A total of 60 sequences located within the HEV SHSW-zs1 genome (GenBank: EF570133] (44 between nt 6104 and nt 6256, and 16 between nt 6360 and nt 6509) together with five full-length swine and human HEV genomic sequences, all derived from Shanghai, were retrieved from GenBank as of June, 2011. All the sequences were aligned with Clustal W (version 1.8), and the sequence percent identity was calculated using Lasergene (version 7.10; DNAstar). Phylogenetic trees were constructed by the neighbor-joining method [10], based on the partial nucleotide sequences of the ORF2 region. Bootstrap values were determined on 3,000 resamplings of the data sets [11]. The criteria used to define HEV genotypes or subtypes were adopted from Lu et al. [12]. These authors demonstrated that an assemblage of 300-450 nucleotides at the 5 ' end of the HEV ORF2 region was highly conserved and that phylogenetic analysis based on this region provided accurate information about the genetic relationships between the HEV isolates and their evolutionary state. The accession numbers of HEV reference sequences are shown in Figure 1

Phylogenetic analysis indicated that six different HEV subtypes, i.e. $3 \mathrm{~b}, 4 \mathrm{a}, 4 \mathrm{~b}, 4 \mathrm{~d}, 4 \mathrm{~h}$ and $4 \mathrm{i}$ (Figure 1) have so far been prevalent in Shanghai. Genotype 4 was the most highly represented genotype among the 65 Shanghai sequences, and subtype $4 \mathrm{i}(29 / 65)$, followed by $4 \mathrm{~d}(10 / 65)$ and $4 \mathrm{~h}(9 / 65)$, the most prevalent subtypes. Of the 29 sequences classified as subtype $4 \mathrm{i}$, four were of human origin and 25 were from swine. HEV strain SH-SW-zs1 [GenBank: EF570133], originating from swine and regarded as the Shanghai prototype HEV strain, belonged to subtype 4i. Furthermore, all the subtype 4i HEV strains so far reported in China were isolated in the eastern part of the country, with $70 \%$ emanating from Shanghai, suggesting that subtype $4 \mathrm{i}$ strains are perhaps indigenous to the municipality. Also included among this subtype was the HEV strain, E067-SIJ05C [GenBank: AB369690], collected from a patient suffering from acute hepatitis E in Japan who had traveled to Shanghai before the onset of the disease. Furthermore, the partial sequence [GenBank: EU034710] of another subtype 4i strain isolated from a human source in Shanghai was found to be virtually identical to a partial HEV sequence [GenBank: EU034714] obtained from a sample of swine serum in eastern China during the same period, suggesting that the isolates had a common origin [13]. To date, a total of nine subtype 4i HEV strains identified from full genomic sequences deposited in GenBank have emanated either from Japan or from Shanghai and neighboring Jiangsu Province. HEV strains of human origin emanating from Japan (JYN-Shiz08L and JKS-Shiz07L) and from Shanghai (E067-SIJ05C) were closely related to HEV strains isolated from wild boar (wbJGF08-1) $[14,15]$ and from swine (SAAS-FX17) [16], respectively. In addition, when phylogenetic clustering of subtype 4i HEV strains was examined (data not shown), sequences from Japan and Shanghai were positioned on different subtype $4 \mathrm{i}$ branches, suggesting that each of these two groups of strains derived from different origins. When compared with other HEV subtype 4 strains across the whole genome, only two specific amino acid substitutions were identified within the ORF1 of subtype 4i. One substitution was located in the methyltransferase motif and the other in the macro domain and might therefore influence HEV replication [17].

In this study, only subtypes $4 \mathrm{~h}, 4 \mathrm{i}$ and $4 \mathrm{~d}$ were found in both swine and humans, whereas subtypes $4 \mathrm{a}$ and $4 \mathrm{~b}$ were confined to either human or swine populations, respectively. According to Zhu et al. [16], subtypes 4a and $4 \mathrm{~b}$ HEV were capable of infecting both humans and swine. Subtype 4a HEV is widely distributed among both the swine and human populations in China as a whole but swine subtype 4a HEV has not been identified in Shanghai. A human subtype 4a strain isolated in Shanghai, JYI-ChiSai01C [GenBank: AB197674], showed the highest nucleotide similarity $(94.0 \%)$ with a swine HEV strain Ch-S-1, from Jilin Province [GenBank: EF077630], suggesting that 4a subtypes in Shanghai were also zoonotic. Human subtype $4 \mathrm{~b}$ HEV has a more limited distribution in China than the swine counterpart [16] and, so far, no human subtype 4b strain has been reported in Shanghai. However, not every sample from hepatitis E cases in Shanghai has been analyzed sufficiently and the possibility that human subtype $4 \mathrm{~b}$ exists among the population of the municipality cannot be excluded.

Genotype $3 \mathrm{HEV}$ strains were geographically limited to Shanghai and neighboring provinces, and seven sequences were classified into subtype $3 \mathrm{~b}$. No human genotype $3 \mathrm{HEV}$ sequences were among those from Shanghai although one strain, EChN22 [GenBank: HM439285], was reported recently in neighboring Jiangsu Province [18]. The EChN22 isolate clustered closely with Shanghai swine isolate FJ527832 and shared 97.2\% nucleotide and $99.6 \%$ amino acid homologies, which suggested that genotype 3 strains prevalent in Shanghai possibly participated in human-swine transmission. 

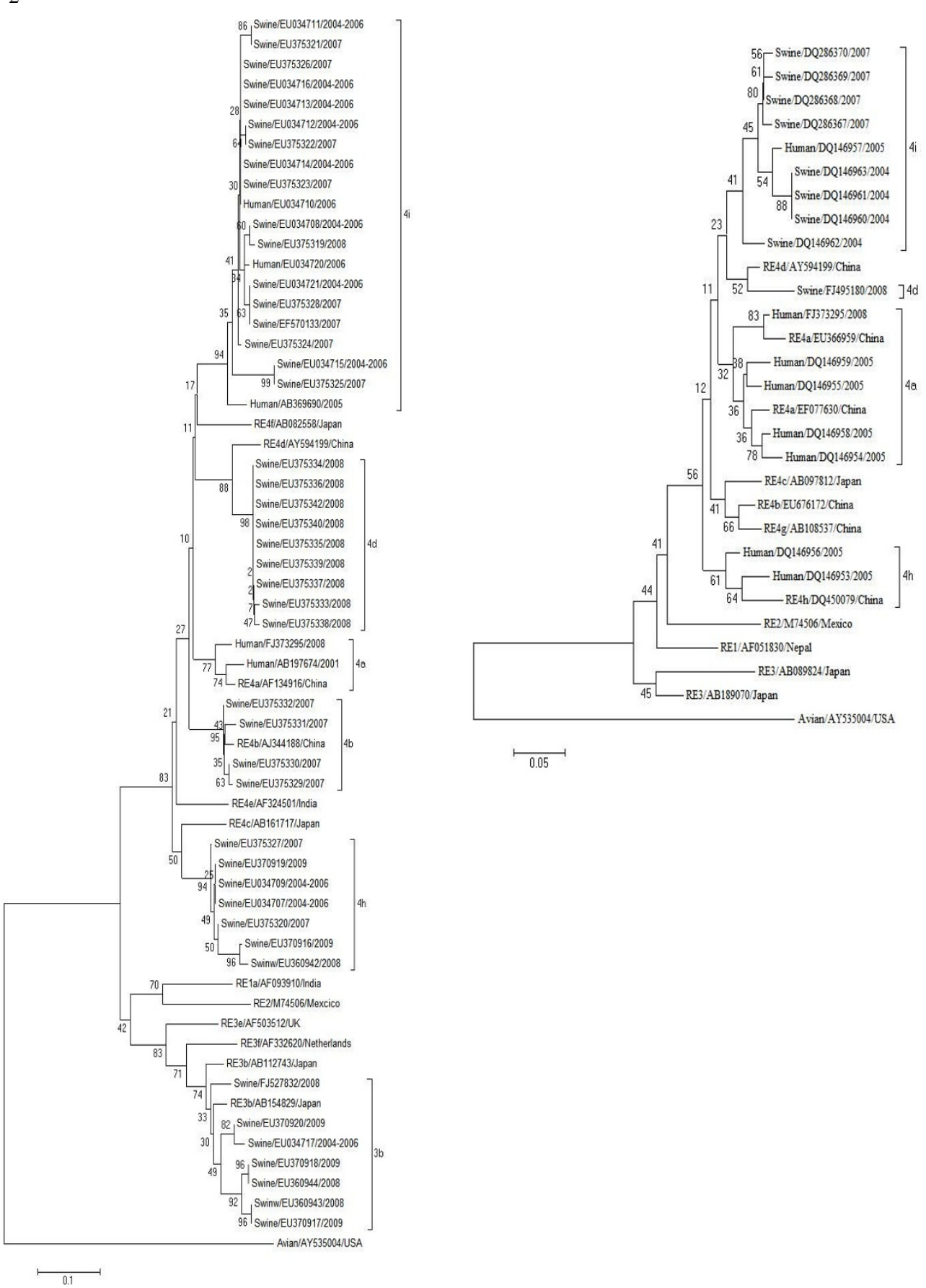

Figure 1 Phylogenetic tree depicting genotypic/subgenotypic status of swine and human HEV strains isolated in Shanghai. A) Analyses based on 5 full-length swine and human HEV genomic sequences and 44 ORF2 sequences located between nt 6,104 and nt 6,256 within the HEV SH-SW-zs1 genome [GenBank:EF570133]. B) Analyses based on 16 sequences located between nt 6,360 and nt 6,509 within the HEV SH-SWzs1 genome. Percent bootstrap support is indicated at each node. For each phylogeny, HEV subtypes were indicated on the outside of the square brackets that define the HEV subtypes. Each branch is labeled with the host of the HEV isolate, GenBank accession number and the year the strain was isolated. Reference sequences are labeled with the prefix RE, the subtype of the sequence, the GenBank accession number and the geographical source. 
No genotype $1 \mathrm{HEV}$ representatives were found in this study, possibly due to the limited number $(10 / 65)$ of human HEV sequences available.

In summary, six different HEV subtypes, i.e. 3b, 4a, $4 \mathrm{~b}, 4 \mathrm{~d}, 4 \mathrm{~h}$ and $4 \mathrm{i}$, have so far been shown to be prevalent in Shanghai, with genotype 4 and subtype $4 \mathrm{i}$ the dominant forms. Only subtypes $4 \mathrm{~h}, 4 \mathrm{i}$ and $4 \mathrm{~d}$ were found in both swine and human hosts, whereas subtypes $4 \mathrm{a}$ and $4 \mathrm{~b}$ were confined to either human or swine populations, respectively. A recent epidemiological investigation has shown that the average incidence of HEV RNA positives among the swine population of Shanghai was approximately $20.0 \%$, and that serum IgG positives reached $72.18 \%$ [19]. Hepatitis infection rates (all types) ranged between 15.0-35.0\% among the younger population and reached $47.0 \%$ in older people. Furthermore, 39.4-69.7\% cases of sporadic hepatitis E involved co-infection with other hepatitis viruses [20]. Human hepatitis E infections have increased significantly in recent years and, in Shanghai, have now become the third most likely cause of hepatitis disease after hepatitis A and B [21]. Consequently, more molecular epidemiology investigations of HEV among swine, and particularly human, populations should be undertaken to assist in HEV control and prevention.

\section{List of abbreviations}

HEV: Hepatitis E virus; ORF: Open reading frame

\section{Acknowledgements}

We thank Dr John Buswell, Institute of Edible Fungi, for linguistic revision of the manuscript. This study was supported by grants from the Shanghai Municipal Natural Science Foundation (No. 09ZR1428100) and the Shanghai Academy of Agricultural Sciences Fund for Young Scientists (2009(16)).

\section{Author details}

1 Institute of Animal Science and Veterinary Medicine, Shanghai Academy of Agricultural Sciences, Shanghai 201106, China. ${ }^{2}$ Shanghai Key Laboratory of Agricultural Genetics and Breeding, Shanghai 201106, China. ${ }^{3}$ Nanjing Agricultural University, Nanjing, Jiangsu 210095, China. ${ }^{4}$ Center for Molecular Medicine and Infectious Diseases, Department of Biomedical Sciences and Pathobiology, College of Veterinary Medicine, Virginia Polytechnic Institute and State University, Blacksburg, VA 24061-0913, USA. ${ }^{5}$ Jiangxi Agricultural University, Nanchang, Jiangxi 330045, China.

\section{Authors' contributions}

YMZ, FYS, RSY, YSZ and ZL participated in the study design, YMZ, SJD and FSS performed the sequence analysis, and all authors participated in writing and revising the manuscript. All authors have read and approved the final version.

\section{Competing interests}

The authors declare that they have no competing interests.

Received: 7 September 2011 Accepted: 15 December 2011 Published: 15 December 2011

\section{References}

1. Chandra VTS, Kalia M, Jameel S: Molecular biology and pathogenesis of hepatitis E virus. J Biosci 2008, 33:451-464.

2. Emerson SU, Anderson D, Arankalle A, Meng XJ, Purdy M, Schlauder GG, Tsarev SA: Hepevirus. In Virus taxonomy VIIIth report of the ICTV. Edited by:
Fauquet CM, Mayo MA, Maniloff J, Desselberger U, Ball LA. London:Elsevier/ Academic Press; 2004:851-855.

3. Teo CG: The two clinico-epidemiological forms of hepatitis E. I Viral Hepat 2007, 14:295-297.

4. Johne R, Plenge-Bönig A, Hess M, Ulrich RG, Reetz J, Schielke A: Detection of a novel hepatitis E-like virus in faeces of wild rats using a nested broad-spectrum RT-PCR. J Gen Virol 2010, 91:750-758.

5. Takahashi M, Nishizawa T, Sato H, Sato Y, Jirintai, Nagashima S, Okamoto H: Analysis of the full-length genome of a hepatitis $\mathrm{E}$ virus isolate obtained from a wild boar in Japan that is classifiable into a novel genotype. $J$ Gen Virol 2011, 92:902-908.

6. Li RC, Ge SX, Li YP, Zheng YJ, Nong Y, Guo QS, Zhang J, Ng MH, Xia NS Seroprevalence of hepatitis E virus infection, rural southern People's Republic of China. Emerg Infect Dis 2006, 12:1682-1688.

7. Li Z, Yu SS, Dong SJ, Zhu YM, Si FS, Shen SY, Jiang ZQ, Yu RS, Zou SX: Reduced prevalence of genotype $3 \mathrm{HEV}$ in Shanghai pig farms and hypothetical homeostasis of porcine HEV reservoir. Vet Microbiol 2009, 137:184-189.

8. Geng J, Wang L, Wang X, Fu H, Bu Q, Liu P, Zhu Y, Wang M, Sui Y, Zhuang H: Potential risk of zoonotic transmission from young swine to human: seroepidemiological and genetic characterization of hepatitis $\mathrm{E}$ virus in human and various animals in Beijing, China. J Viral Hepat 2011 18:e583-590.

9. Zhu YM, Dong SJ, Si FS, Yu RS, Yu XM, Zou SX, Li Z: Swine and human hepatitis E virus (HEV) infection in China. J Clin Virol 2011, 52:155-157.

10. Saitou N, Nei M: The neighbor-joining method: a new method for reconstructing phylogenetic trees. Mol Biol Evol 1987, 4:406-425.

11. Felsenstein J: Confidence limits on phylogenies: an approach using the bootstrap. Evolution 1985, 783-791.

12. Lu L, Li C, Hagedorn CH: Phylogenetic analysis of global hepatitis E virus sequences: genetic diversity, subtypes and zoonosis. Rev Med Virol 2006, 16:5-36.

13. Zhang W, Shen Q, Mou J, Gong G, Yang Z, Cui L, Zhu J, Ju G, Hua X: Hepatitis $\mathrm{E}$ virus infection among domestic animals in eastern China. Zoonoses Public Hlth 2008, 55:291-298.

14. Sato Y, Sato H, Naka K, Furuya S, Tsukiji H, Kitagawa K, Sonoda Y, Usui T, Sakamoto H, Yoshino S, Shimizu Y, Takahashi M, Nagashima S, Jirintai, Nishizawa T, Okamoto H: A nationwide survey of hepatitis $\mathrm{E}$ virus (HEV) infection in wild boars in Japan: identification of boar HEV strains of genotypes 3 and 4 and unrecognized genotypes. Arch Virol 2011, 156:1345-1358

15. Takahashi K, Kitajima N, Abe N, Mishiro S: Complete or near-complete nucleotide sequences of hepatitis $E$ virus genome recovered from a wild boar, a deer, and four patients who ate the deer. Virology 2004 330:501-505.

16. Zhu YM, Yu XM, Si FS, Zhang YS, Zou SX, Li Z: Full-genome sequence analysis of a hepatitis E virus (HEV) strain SAAS-FX17 and potential disease severity determinants identification between genotype 3 and genotype 4 HEV strains. Virology 2011, submitted.

17. Ahmad I, Holla RP, Jameel S: Molecular virology of hepatitis E virus. Virus Res 2011, doi:10.1016/j.virusres.2011.02.011

18. Yan Y, Zhang W, Shen Q, Cui L, Hua X: Prevalence of four different subgenotypes of genotype 4 hepatitis $E$ virus among swine in the Shanghai area of China. Acta Vet Scand 2008, 50:12.

19. Zhou JP, Sun QY, Liu PH, Xue X, Li KH, Lu J, Zhang WY, Qi XY: Serological investigation on hepatitis $\mathrm{E}$ of several animal species in Shanghai. Prog in Vet Med 2006, 27:85-88, (Article in Chinese).

20. Li YT, Zhu YY, Shen WG, Zhang AX, Zhang JM, Ren H, Yuan GP, Gu L: The analysis of HEV genotypes isolated from sporadic acute hepatitis $E$ in Shanghai. Virologica Sinica 2002, 17:106-109.

21. Kang $L Y$, Pan QC, Jin ZC, Zhou TK, Fang H, Xue YL, Zhang W, Jiang $Q$, Zhu QR, Yao GB, Zhang JM, Zheng XH: The molecular investigation of hepatitis C and E virus infection in Shanghai. J Prev Med 1995, 7:438-441.

doi:10.1186/1743-422X-8-541

Cite this article as: Zhu et al:: Molecular epidemiology of hepatitis $\mathrm{E}$ virus infections in Shanghai, China. Virology Journal 2011 8:541. 